\title{
Interférences
}

Ars scribendi

$6 \mid 2012$

La question de la littérarité

\section{Les mille traces de la conscience littéraire dans les Métamorphoses d'Ovide}

\section{Anne Videau}

\section{(2) OpenEdition}

1 Journals

Édition électronique

URL : http://journals.openedition.org/interferences/176

DOI : 10.4000/interferences. 176

ISSN : $1777-5485$

Éditeur

HiSoMA - Histoire et sources des Mondes antiques

Édition imprimée

Date de publication : 1 janvier 2012

ISSN : 1777-5485

\section{Référence électronique}

Anne Videau, « Les mille traces de la conscience littéraire dans les Métamorphoses d'Ovide »,

Interférences [En ligne], 6 | 2012, mis en ligne le 11 décembre 2014, consulté le 15 septembre 2020.

URL : http://journals.openedition.org/interferences/176 ; DOI : https://doi.org/10.4000/interferences. 176

Ce document a été généré automatiquement le 15 septembre 2020.

Tous droits réservés 


\title{
Les mille traces de la conscience littéraire dans les Métamorphoses d'Ovide
}

\author{
Anne Videau
}

1 En préférant au terme de "littérarité » l'expression plus archaïque de «conscience littéraire», qui implique autant l'auteur que le destinataire, il est loisible de faire littéralement l'« investigation ", de suivre à la trace la manière dont Ovide marque le texte des Métamorphoses comme texte, comment il le met en perspective en introduisant une place pour le lecteur pour cette mise en perspective et en lui en offrant la jouissance. Ce sont les divers procédés de cette mise en abyme que nous considérerons d'emblée comme sa "littérarité » et qui nous guideront. Certains aspects ont pu en être dessinés depuis les années quatre-vingt-dix, spécialement par Alessandro Barchiesi et Gianpiero Rosati, Perrine Galland, Isabelle Jouteur et Gilles Tronchet. Les études de Jean-Christophe Jolivet sur l'allusion dans les Héroïdes et celles d'Alain Deremetz sur la réflexivité dans la poésie latine offrent des instruments transposables à l'œuvre épique du poète de Sulmone. Nous nous limitons ici à deux versants, qui touchent l'épopée en tant que poème narratif et/ou narration poétique : la mise en perspective ovidienne de l'élaboration de la narration et sa représentation distanciée des signifiants.

\section{Mise en perspective de l'élaboration de la narration}

2 On se souvient de quelle manière Aristote, au chapitre 3 de la Poétique ${ }^{1}$, fait de la prise de parole du héros Ulysse lui-même une des caractéristiques éminentes de l'épopée héroïque et un élément de la supériorité de l'odyssée, l'épopée apparaissant comme un mixte de narration à la $3^{\mathrm{e}}$ et à la $1^{\text {re }}$ personne, qui combine les prises de parole d'un narrateur "extradiégétique et hétérodiégétique » omniscient (le narrateur premier raconte une histoire dont il n'est pas un participant) et d'un narrateur « intradiégétique et homodiégétique » (narrateur-héros qui raconte sa propre histoire à 
tels personnages), ainsi Ulysse aux Phéaciens ${ }^{2}$. Du point de vue de l'élaboration de la narration, les Métamorphoses se caractérisent - c'est l'aspect auquel Gilles Tronchet a consacré sa Métamorphose à l'œuvre: recherches sur la poétique d'Ovide dans les Métamorphoses d'Ovide ${ }^{3}$ - par une démultiplication à l'extrême des récits enchâssés et des voix narratives.

3 Le «mélange des genres » a été également repéré naguère (1924) par Wilhelm Kroll ${ }^{4}$ comme un des traits consistants de la conscience littéraire. Pour cette étude, concernant Ovide, les ouvrages de Georges Lafaye, Les Métamorphoses d'Ovide et leurs modèles grecs ${ }^{5}$ (1904), et d'Isabelle Jouteur, Jeux de genre dans les Métamorphoses d'Ovide (2001) ${ }^{6}$, sont des bases indispensables.

\section{Jeux de genres et mise en abyme des récits}

4 Parmi les possibilités multiples du poème des Métamorphoses, nous avons donc distrait un épisode de leur premier Livre qui joue du côté de la «fonction métalinguistique " (le code lui-même devient objet du message) en conjoignant problématisation du «mélange des genres » et mise en abyme du récit.

Mercure a été chargé par Jupiter d'éliminer le monstre Argus, qui surveille son amante Io métamorphosée en génisse pour le compte de sa femme Junon (I, 676-684) :

Hac agit ut pastor per deuia rura capellas

dum uenit adductas et structis cantat auenis

Voce nou captus custos Iunonis et arte:

'Quisquis es hoc poteras mecum considere saxo'

Argus ait 'neque enim pecori fecundior ullo [680]

herba loco est aptamque uides pastoribus umbram'

Sedit Atlantiades et euntem multa loquendo

detinuit sermone diem iunctisque canendo

uincere harundinis seruantia lumina temptat

[De sa baguette], il chasse devant lui, tel le berger, à travers champs, hors les

chemins frayés,

les chevrettes qu'il a avec lui amenées, et sur les chalumeaux compactés, il module.

Ravi par le son inouï, le gardien de Junon, et son art :

'Qui que tu sois, tu aurais pu avec moi t'asseoir sur cette pierre',

dit Argus, 'Nulle part troupeaux ne trouvent [680]

herbe plus abondante et tu vois là une ombre qui convient aux bergers'.

L'Atlantiade s'est assis et à longuement discourir, il suspend

le décours du jour, en jouant sur ses roseaux

unis, il s'efforce de vaincre la vigilance de ses yeux.

Dans ce passage, on admire d'abord la mise en perspective du mélange des genres par la reprise littérale d'éléments empruntés à la bucolique.

6 Avec un lexique tout virgilien, Ovide campe Mercure en persona bucolique, pastor de capellas, dans un décor typique de rura ; il introduit une situation d'élocution non moins typique du chant amébée au moment où Argus invite le dieu-chevrier à s'asseoir près de lui, tout comme Ménalque le fait pour Mopsus dans la cinquième Bucolique ${ }^{7}$, et sous l'ombre protectrice, tel Tityre dans la première. Le lecteur retrouve la double articulation bucolique entre la parole et la musique (parole: multa loquendo, et modulation musicale : structis cantat auenis ; iunctisque canendo [...] harundinis). C'est, en effet, à la fois de la sonorité, uoce noua, et de l'art, noua [...] arte, tous deux "nouveaux », 
de la flûte dite de Pan, iunctisque [...] harundinis, qu'est narrée l'origine dans ce récit enchâssé.

Le dieu rivalise avec le monstre pour le "vaincre », uincere, le verbe retrouvant son sens guerrier, épique, à la fin de l'épisode, à partir du sens agonistique bucolique.

On admirera aussi le jeu sur les voix narratives.

Le narrateur principal abandonne la parole à Mercure ${ }^{8}$, qui amorce l'étiologie de l'instrument, dont il joue hic et nunc, la flûte syrinx. Mais il la lui reprend presque aussitôt : à cet instant raconté où le dieu Pan s'apprête à adresser à la nymphe Syrinx sa sollicitation amoureuse (v. 700-701) ${ }^{9}$ et jusqu'à la métamorphose en roseaux de celleci, puis sa transformation en flûte. Et ce narrateur principal conclut : «Voilà ce qu'allait dire le Cyllénien quand il vit que tous / les yeux [du monstre] s'étaient fermés... ", omnes / succubuisse oculos (v. 713-714) ${ }^{10}$.

Pourquoi cette «subtilisation» de la fin de l'histoire au narrateur secondaire, Mercure?

Ovide a inséré l'aventure de Pan avec Syrinx au sein de la grande histoire d'Io, inspirée des tragiques (Eschyle, Les Suppliantes et Prométhée enchaîné, Sophocle, Inachus perdu). Or, celle-ci succède au premier érôtikon pathéma de l'épopée : la "passion d'amour " d'Apollon pour Daphné. Trois éléments narratifs, voire "mythèmes" - l'échec amoureux de Pan, dieu de l'Arcadie originelle, la métamorphose végétale de Syrinx, qui est chasseresse, en roseaux -, font de l'histoire contée par Mercure un doublet plus léger, voire satyrique, de la passion malheureuse primordiale d'Apollon pour Daphné, elle-même émule de Diane chasseresse, et muée, elle, en laurier.

La subtilisation du dénouement de l'histoire à Mercure viendrait exhiber le travail narratologique opéré par l'auteur. Le «vol» subi par le dieu/prince des voleurs, au profit d'un abrégé mythologique sans vergogne, signifie plaisamment au lecteur que si Mercure avait conté in extenso, il y aurait eu là une répétition, qui aurait bien pu... l'endormir, lui, lecteur dont Argus est un relai, une figure incluse dans le récit. Ovide dénude donc son procédé de composition narrative: le tissage, au-delà de la « rhapsodie »..., sur les structures analogues de ce que nous appelons « mythes». L'épisode de rivalité à la toile entre Arachné et Pallas, au Livre VI, par exemple, met clairement en abyme cette pratique, puisque en particulier Arachné bâtit la sienne sur la répétition du schéma du rapt amoureux des héroïnes par les dieux olympiens :

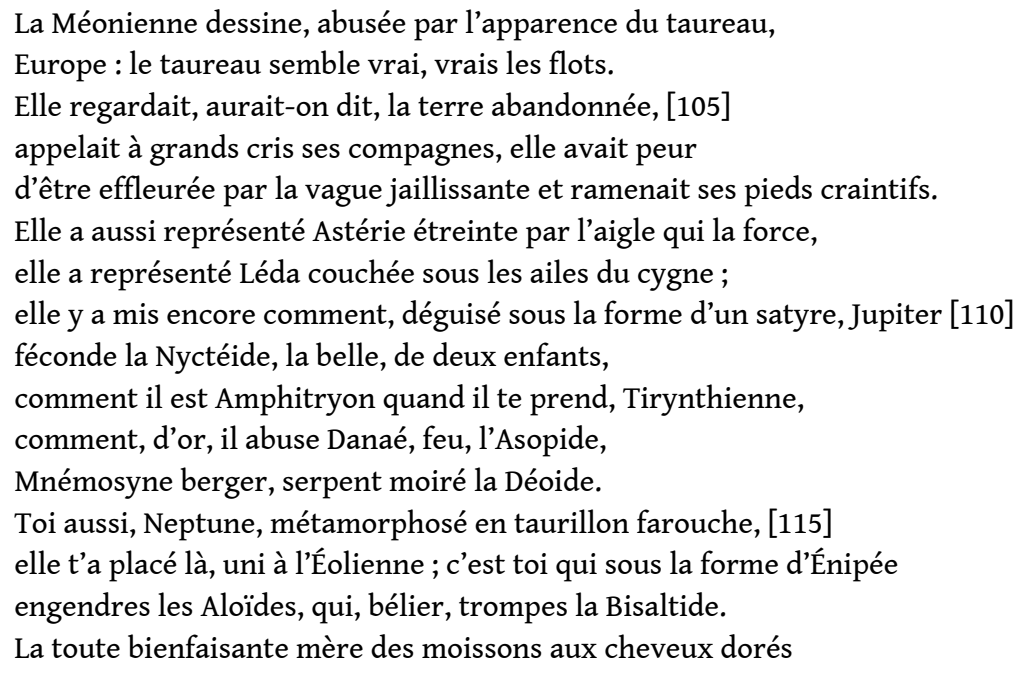


t'a senti cheval, elle t'a senti oiseau, la mère du cheval ailé

aux cheveux de serpents, Mélantho t'a senti dauphin. [120]

De tous, elle a rendu exactement la forme,

et la forme des lieux. Il y a Phébus sous la figure d'un paysan,

puis comment il revêt tantôt les plumes d'un épervier, et tantôt la cambrure

d'un lion, comment, berger, il a trompé la Macaréide Issé,

comment Liber abuse en grappe fallacieuse Érigone, [125]

comment Saturne, cheval, engendre Chiron à double forme.

14 Vis-à-vis de la macrostructure narrative de l'épopée et de la hiérarchie des genres poétiques, le poète souligne enfin la signification primordiale de l'érôtikon pathéma princeps qui a donné le jour à Laurus, «Laure », à l'emblème qui couronne la chevelure et la lyre de Phébus-Apollon, à l'emblème de toute poésie. Par l'amenuisement du récit qui concerne Syrinx, Ovide signifie que si la naissance de la poésie ${ }^{11}$ dans l'érôtikon pathéma apollinien est première, la poésie bucolique, elle, en est un "genre", sans doute originel, en Arcadie, mais... secondaire, subordonné, mineur.

\section{L'autonomisation de la description comme " morceau esthétique » : deux modes d'articulation de la description avec le récit}

L'autonomisation de la description comme «morceau esthétique » peut-elle aussi être reconnue comme marque de "littérarité " - c'est ce que suggère F. Frazier -, en ce qu'elle relèverait de l'«affirmation du caractère intransitif, non instrumental de l'œuvre », pour reprendre une formule de Tzvetan Todorov ${ }^{12}$ ?

16 En ce sens, la comparaison entre la lutte de Laoocon contre les serpents envoyés par Neptune, dans l'Énéide, au Chant II, entre tous illustre et fondateur pour la réflexion sur les arts, et le combat du héros Cadmus, au Livre III des Métamorphoses ${ }^{13}$, contre le serpent de la source de Dircé en Béotie ${ }^{14}$, démontre la divergence entre la pratique virgilienne de la description dans son rapport avec la narration - pratique aristotélicienne, en quelque manière « classique » - et celle d'Ovide. Nous nous sommes appuyée naguère de façon très stricte sur une stylistique de la syntaxe et de la morphologie, spécialement sur l'étude de l'usage des ablatifs, ablatifs dits absolus et ablatifs instrumentaux et/ou ablatifs dits "de qualité " ${ }^{15}$, sur l'étude contrastée de l'usage des participes et des qualifiants, sur l'usage de la forme médio-passive chez Ovide, pour dégager les traits distinctifs de leurs deux esthétiques.

Chez Virgile, soutenons-nous :

Là même où la description semblerait pouvoir prendre de l'autonomie, où la valeur de l'ablatif [...] ouvrirait sur une perspective qualifiante et non pas narrative, c'est la valeur du cas par rapport à l'action qui est présente. [...] Aux adjectifs qualificatifs qui signifient un état des êtres et des choses, Virgile préfère des participes, qui, participes présents, décrivent un procès, ou, participes parfaits, immobilisent un procès en un état qui conserve cependant la marque de ce procès. [...] Ainsi la description ne prend quasiment aucune autonomie par rapport à la narration d'action. Autrement dit, Virgile applique [à la relation entre description et narration] la règle aristotélicienne [qui concerne] l'épisode [lequel, selon le philosophe] doit avoir, pour que la narration soit « une », unifiée, une dépendance de cause ou de conséquence par rapport à l'intrigue.

$\mathrm{Ce}$ avec une correspondance de fonctionnement entre microstructure et macrostructure.

La répétition du verbe sinuo d'un poème à l'autre permet de mettre en évidence la différence des visées. Virgile écrit à l'actif, avec sujet, objet et instrumental 
distincts : sinuatque immensa uolumine terga (v. 208), « [le reste de leur corps] courbe en son orbe leur échine immense » et [...] ipsum [...] / corripiunt spirisque ligant ingentibus (v. 216-217), « ils le saisissent et l'attachent dans leurs spirales énormes ». Ovide reprend: et immensos saltu sinuatur in arcus (v. 42), "et, bondissant, il se courbe en arcs immenses », et, dans la seconde parade, immensum spiris facientibus orbem cingitur (v. 77), «Formant de ses spirales un orbe immense, il se referme sur lui-même ». Dans les deux cas, il choisit au lieu d'une forme active un médio-passif qui décrit le retour du serpent sur lui-même, un procès en retour, et non sa prise sur l'adversaire. Il met donc en évidence non l'acte, mais la forme.

Nous avons pu conclure :

De Virgile à Ovide, on transite donc d'une esthétique de l'émotion, dépendante de la construction du mythos, à une esthétique du spectacle. On sait comment, dans la Poétique, Aristote mettait de côté les éléments opsis, "spectacle", et mélopoïa, "musique », pour privilégier tragédie et épopée fondées sur l'agencement du mythos avec péripétie et reconnaissance ${ }^{16}$, dans une esthétique de l'émotion intellectuelle. Virgile se situe exactement dans cette perspective. Ovide en revanche tend vers une esthétique qui accorde en quelque sorte place à opsis et mélopoïa.

Cette conclusion amène au second versant de notre investigation actuelle.

\section{Conception et représentation de la conception du signifiant dans les Métamorphoses} conception et la représentation de la conception du signifiant par Ovide dans les Métamorphoses. avec la «fonction poétique » qui met l'accent sur le «message » lui-même, le jeu sur le signifiant ne saurait être considéré en soi comme index de «littérarité »: quand Jakobson définit la «fonction poétique ", il en emprunte comme on sait l'exemple à la campagne politique d'Eisenhower de 1952, dont le slogan était fondé sur la paronomase: I like Ike; le citoyen ne pouvait qu'aimer cet Ike dont le nom était intrinsèque à like, l'amour ${ }^{18}$. Au point que Georges Mounin a pu écrire que :

la fonction poétique chez Jakobson explique aussi bien (ou mieux) les slogans comme I like Ike que Spleen de Baudelaire ${ }^{19}$.

En revanche, il est patent qu'Ovide désigne à une lecture « littéraire » " poéticienne » la totalité de la «surface » de son texte ${ }^{20}$.

21 Sa sensibilité au choix des signifiants et à la signification de ce choix s'illustre en effet de manière fondamentale dans un passage remarquable du Livre XI : l'épisode des époux de Trachyne, Céyx et Alcyone, séparés par la tempête meurtrière qui engloutit le héros. Junon, qu'Alcyone ne cesse de supplier pour son retour, décide de lui envoyer un rêve qui la prépare à comprendre l'issue fatale. Sollicité par Iris, messagère de la déesse, le Sommeil éveille alors trois de ses fils polymorphes, Morpheus qui mime les humains, Phantasos, qui imite les objets inanimés, et, en deuxième, celui qui, écrit-il (v. 639-641):

fit fera, fit uolucris, fit longo corpore serpens

Hunc Icelon Superi mortale Phobetora uulgus [640]

nominat..

se fait bête, se fait oiseau, se fait serpent au long corps.

Lui, Ceux-d'en haut, c'est Icélon, la foule des mortels, Phobétôr [640]

qu'ils le nomment ${ }^{21}$... 
Ikelon, ici neutre substantivé de l'adjectif grec, « l'identique », caractérise au chant X de l'Odyssée la couleur de la fleur moly donnée par Hermès à Ulysse, que le héros compare au « lait », heikelon galakti :

Ayant ainsi parlé, l'Éblouissant me donna l'herbe

qu'il avait déterrée et me décrivit sa nature.

Sa racine était noire et sa fleur pareille au lait pur ;

les dieux l'ont appelée moly et les mortels

ont peine à l'arracher ; mais les dieux peuvent tout ${ }^{22}$.

Ovide remémore au lecteur la filiation homérique de son propre poème: les deux épopées homériques connaissent cette double nomination ; outre le passage cité, chez Alcinoos, elle apparaît pour les Roches planctes (Od. XII, 59-61), pour Égéon/Briarée (Il. I, 403-404), pour Batiée/Myrine (Il. II, 811-814) ${ }^{23}$.

En même temps, par cette réminiscence, il marque le souci de la nomination qui, dans le passage cité :

Hunc Icelon Superi ${ }^{\mathrm{P}}$ mortale Phobetora uulgus

s'inscrit, à travers le rejet du verbe (sur le vers 641), nominat, et le soulignement disjonctif de la penthémimère qui sépare et spécifie Superi vs mortale uulgus.

Le bel ouvrage d'Anagrammes et allitérations ${ }^{24}$ de Françoise Bader étudie ces passages homériques de la langue des dieux comme "l'inventaire" d'une langue sacralisée, l'héritage originel de l'épopée en tant que savoir sur les lettres et les noms.

Frederick Ahl, dans son Metaformations: Soundplay and Wordplay in Ovid and Other Classical Poets, n'a pas été le premier, en 1985, à focaliser l'interprétation des Métamorphoses sur des réseaux de signifiants. Il a été précédé (et il le suggère) dans cette voie par... Ferdinand de Saussure. Car, si l'on connaît bien, pour ce que Jean Starobinski les a remis à l'honneur depuis 1964, suivi de bien d'autres, les Cahiers Lucrèce du grand linguiste ${ }^{25}$, les trois numéros que ce dernier a consacrés à Ovide restent encore inexploités dans le fonds genevois. On sait comment Saussure cherchait à mettre au jour la manière dont les poètes anciens auraient codé de façon sacrée leurs textes en les construisant par diffraction d'un nom sur des unités poétiques déterminées.

La "littérarité » des Métamorphoses d'Ovide gît donc aussi et peut-être d'abord dans la mise en abyme constante du signifiant.

Quelques épisodes majeurs sollicitent fortement cette interrogation : nous en évoquons ici deux qui nous sont spécialement chers, sous des angles renouvelés. Nous avons été attentive naguère à l'histoire qui met en scène la nymphe Écho avec le jeune Narcisse. Ovide s'y intéresse au phénomène physique de réflexion sonore en l'associant au phénomène de réflexion visuelle au cœur de cette grande histoire de la physis que sont les Métamorphoses. Ce contrepoint imago sonore-imago visuelle, écrivent Françoise Frontisi Ducroux et Jean-Pierre Vernant :

est déjà en germe dans les théories scientifiques des présocratiques. Il est explicite dans un passage capital du Phèdre [...]. Et il est largement développé par Aristote, qui explique la production de l'écho par analogie avec la réflexion de la lumière. Le son, explique le philosophe, est toujours renvoyé en écho, et la lumière, de même, se réfléchit toujours, produisant une ombre-reflet, la skia, aux valeurs équivoques ${ }^{26}$.

Ovide mime le phénomène de réflexion sonore à la fois par la structuration des groupes dans les vers et par les assonances, dès le moment où la nymphe s'éprend du jeune homme (v. 356-361) :

Adspicit hunc trepidos agitantem in retia ceruos uocalis nymphe qua nec reticere loquenti

Interférences, 6 | 2012 
nec prior ipsa loqui didicit resonabilis Echo

Corpus adhuc Echo non uox erat et tamen usum

garrula non alium quam nunc habet oris habebat [360]

reddere de multis ut uerba nouissima posset ${ }^{27}$.

Dans la périphrase qui la dénomme (v.357-358), le syntagme initial, en tête de vers, uocalis nymphe (v. 356), et le syntagme ultime, en fin de vers, resonabilis Echo, enserrent dans leur résonance inversée, anagrammatique : uocalis vs Echo, [ok] vs [ko], les sons latins [ok], [ek], nec reticere loquenti, nec loqui. Cette résonance est répétée, dans l'ordre inverse, au vers suivant, v. 359, autour de la penthémimère : Echo non uox, [ko] vs [ok]. Jusqu'à ce qu'à son dépérissement sous l'effet du mal d'amour qui la ronge, il ne reste plus d'Echo que Vox, aux vers 399-401: Vox manet [...] sonus est qui uiuit in illa.

C'est-à-dire que, dans une lecture triviale, ne demeure de la nymphe jadis charmante plus que "le son ». Mais, en même temps, de cet echo, signifiant de la langue grecque, Ovide a fait passer le lecteur à la uox de la langue latine, à ce «signifiant » uox, celui qui désigne l'écho comme «son » dans la langue latine, autrement dit, il a traduit et saisi cette uox dans sa langue et celle de l'Empire dont les Métamorphoses disent l'extension.

Entre ces deux moments du texte, Ovide aura mis en scène un "simulacre d'échange ", alternce [...] imagine uocis (v. 385), un paraklausithyron impensable, puisque Écho ne peut commencer un discours, qu'elle ne peut s'adresser à un autre :

Forte puer comitum seductus ab agmine fido

dixerat « Ecquis adest ? » et « ... adest » responderat Echo [380]

Hic stupet utque aciem partes dimittit in omnis

Voce « Veni » magna clamat Vocat illa uocantem

Respicit et rursus nullo ueniente : "Quid » inquit

" me fugis? " et totidem quot dixit uerba recepit

Perstat et alternce deceptus imagine uocis [385]

«Huc cœeamus» ait nullique libentius umquam

responsura sono: « ... cœeamus » rettulit Echo

et uerbis fauet ipsa suis egressaque silua

ibat ut iniceret sperato bracchia collo

Ille fugit fugiensque « Manus complexibus aufer [390]

Ante » ait " emoriar quam sit tibi copia nostri»

Rettulit illa nihil nisi : «... sit tibi copia nostri »

Spreta latet siluis pudibundaque frondibus ora

protegit et solis ex illo uiuit in antris

D'aventure l'enfant, entraîné loin de la troupe de fidèles compagnons,

avait dit : « Quelqu'un est là ? ", « ... est là », avait répondu Écho. [380]

Lui, stupéfait, jette les yeux de tous côtés.

Il appelle d'une voix forte : «Viens! » À l'appel, elle renvoie l'appel.

Il regarde en arrière, et, encore, comme il ne vient personne : « Pourquoi donc

me fuis-tu? », dit-il, et autant de mots il a dits, autant il lui en est répliqué.

Il insiste, abusé par le simulacre d'échange : [385]

«Viens, réunissons-nous! », dit-il et, jamais elle n'aurait

aimé davantage répondre, Écho redit : « ... unissons-nous! »

Elle se prend à ses mots et sortant

des bois, elle s'en allait entourer de ses bras le cou tant espéré :

il fuit, et dans sa fuite : «Garde tes mains de ces embrassements, [390]

je mourrai plutôt que tu aies de moi possession. »

Elle n'a rien redit qu'« ... aie de moi possession!"

Méprisée, elle se cache dans les forêts, elle dissimule, honteuse, son visage

sous la feuillée et depuis de temps vit aux antres solitaires ${ }^{28}$. 
ne revenons sur ce moment de syntaxe mimétique virtuose - Ovide produisant au fil du passage une impression systématique analogue au mécanisme du phénomène que pour la pointe des vers 391-392 : sit tibi copia nostri, où le parallélisme à la rime vient exacerber à la fois l'identité et l'inversion des discours, le refus de Narcisse en contexte (ante ... emoriar quam) venant à exprimer le désir d'Écho, la répétition du même énoncé délivrant... le message strictement inverse.

L'histoire, le quasi-roman, de Céphale et Procris, est fondé, du point de vue qui nous intéresse, sur une récurrence phonique, un réseau [aur], [or], de manière tout aussi remarquable. C'est aussi une histoire de uoces, «sons", et de uoces, "noms ", tels que les oreilles, aures, les capteraient... Comme il le raconte, au lever du iubar aureus du soleil (v. 663), le bel Athénien Céphale est enlevé, à son corps défendant chez notre auteur, par Aurora, sur l'Hymette florentis (v. 702) :

... uidet pulsis Aurora tenebris

inuitumque rapit...

... Aurore, au lever des ténèbres me voit

et, malgré moi, m'enlève ${ }^{29}$...

Dans la structure en écho et les figures anagrammatiques des signifiants qui dessinent sa capture totale dans l'amour de sa femme :

Pectore Procris erat Procris mihi semper in ore

$\mathrm{Au}$ cœur, j'avais Procris, toujours Procris à la bouche ${ }^{30}$

e maintes fois un chant équivoque en harmonie avec son attente pressante, que mime l'anaphore du signifiant Aura :

Aura petebatur medio mihi lenis in aestu.

Auram exspectabam. Requies erat illa labori.

'Aura' recordor enim 'uenias' cantare solebam

une chanson parfaitement érotique, dont la destinataire, à ce qu'il chante, est source de la plus haute volupté: Tu mihi magna uoluptas (v. 817), et qui s'achève sur ce vœu (v. 820):

Spiritus iste tuus semper capiatur ab ore

Que toujours ce tien souffle soit par ma bouche accueilli!

qui rime avec le Procris mihi semper in ore, auquel, sans crier gare, il s'est substitué.

Cette articulation renouvelée du nom d'Aura est recueillie par l'aurem d'un quidam (v. 821-822) :

Vocibus ambiguis deceptam praebuit aurem

nescio quis nomenque aurae tam saepe uocatum

esse putat nymphae nympham mihi credit amari

Par ces paroles équivoques trompé, je ne sais qui prêta

l'oreille et/à ce nom si souvent prononcé d'aure

il le croit celui d'une nymphe, pense que je suis amoureux d'une nymphe 
« souffle d'air », porté à l'oreille de Procris, tel qu'entendu :

... lingua refert audita susurra,

... sa langue rapporte les audita sussurra chuchotis entendus [825]

en onomatopée assonante avec aura-aurora. étymologies varronniennes, ainsi le lien entre aura et Aurora : aura vaut pour les vents doux qui émanent de la terre au lever du soleil, à l'aurore (Argonautiques orphiques 338). Le poète Pacuvius cité par Varron $(L . V, 24)$ réunit aura et aurora sous le même trait :

Terra exhalat auram atque auroram humidam

La terre exhale brise et aurore humide

comme chaque fois qu'il met en abyme les jeux de la langue, signifiants, dans la morphologie et la syntaxe du discours, comme il met en abyme les jeux sur les voix narratives et le mélange des genres, le lecteur ne saurait y pressentir aucune gratuité esthétique.

43

ien au contraire, la reconnaissance et l'amour de la "littérarité ", dans les Métamorphoses comme récit et comme poème, la jouissance partagée avec le lecteur à percevoir le «jeu» du texte, ouvrent sur l'interrogation de ce qu'est cette psyché humaine qui ne se constitue que du langage et des ambiguités qui lui sont inhérentes. Il faudrait faire aussi, étonnamment car les poètes latins -comme l'a écrit Françoise Desbordes - ne s'y sont guère intéressés, une place à la lettre, ainsi ce $\mathrm{S}$ du 
serpent, sa sibilation, qui parcourt le poème pour venir le clore, en coronis, comme l'a si magnifiquement montré A. Barchiesi, le $\mathrm{S}$ du Serpent Esculape, fils de la nymphe du même nomen, qui emblématise aussi un pouvoir salvateur nouveau.

Autrement dit, s'il n'y a pas pour Ovide d'autonomie du "littéraire ", alors qu'il témoigne de la conscience la plus aiguë de la «littérarité », c'est pour en désigner la force créatrice et politique, en récusant au passage la frontière tracée par Aristote entre poésie «scientifique » descriptive (Empédocle) et poésie de la mimésis (Homère).

\section{BIBLIOGRAPHIE}

AHL F. 1985, Metaformations: Soundplay and Wordplay in Ovid and Other Classical Poets, Ithaca - London. ARON T. 1984, Littérature et littérarité : un essai de mise au point, Annales littéraires de l'Université de Besançon 292, Paris.

BADER Fr. 1993, Anagrammes et allitérations, Orbis Supplementa 1, Paris - Louvain.

COLlaRT J. (éd.) 1954, De lingua latina. Livre V, Publications de la Faculté des Lettres de Strasbourg 122, Paris.

FRAZIER F. 2009, Poétique et création littéraire en Grèce ancienne : la découverte d'un "nouveau monde ", Institut des sciences et techniques de l'Antiquité, Besançon.

FRONTISI-DUCROUX F., VERNANT J.-P. 1997, Dans l'œil du miroir, Paris.

DUPRIEZ B. 1984, Gradus : les procédés littéraires (dictionnaire), 10-18 1370, Paris.

GANDON F. 2001, De dangereux édifices : Saussure lecteur de Lucrèce : les cahiers d'anagrammes consacrés au De Rerum natura, Bibliothèque de l'information grammaticale 50, Louvain - Paris.

GANDON F. 2003, «Chaos des corps, chora des mots. Onze vers de Lucrèce sur l'illusion amoureuse ", in S. Bouquet (dir.), Ferdinand de Saussure, Cahier de l'Herne 76, p. 430-441.

GANDON F. 2006, Le nom de l'absent : épistémologie de la science saussurienne des signes, Limoges.

JAKOBSON R. 1963, Essais de linguistique générale, traduit et préfacé par N. Ruwet, Paris.

KROLL W. 1924, Studien zum Verständnis der römischen Literatur, Stuttgart.

LAFAYE G. 1971, Les Métamorphoses d'Ovide et leurs modèles grecs, Hildesheim (Bibliothèque de la Faculté des lettres de l'Université de Paris 19, Paris, 1904).

JOUTEUR I. 2001, Jeux de genre dans les Métamorphoses d'Ovide, Bibliothèque d'études classiques 26, Louvain - Paris.

MoUnIN G. 1972, La linguistique du XX $x^{e}$ siècle, Le linguiste 13, Paris.

TODOROV T. 1965, Théorie de la littérature : textes des formalistes russes, Tel quel, Paris.

TODOROV T. 1978, Les genres du discours, Poétique 23, Paris. 
TRONCHET G. 1998, La métamorphose à l'œuvre : recherches sur la poétique d'Ovide dans les Métamorphoses, Bibliothèque d'études classiques 13, Louvain - Paris.

VIDEAU A. 2010, La poétique d'Ovide, de l'élégie à l'épopée des Métamorphoses : essai sur un style dans l'histoire, Rome et ses renaissances, Paris.

\section{NOTES}

1. Arist., Po. 3,1448 a 19 sq.

2. Ainsi qu'a pu les désigner Genette 1969. Tronchet 1998 a examiné certains aspects de la narration ovidienne de ce point de vue avec une grande rigueur.

3. Tronchet 1998.

4. Frazier 2009, p. 16, n. 1 ; Kroll 1924, chap. IX.

5. Lafaye 1971.

6. Jouteur 2001.

7. Verg., Ecl. V, 1-3 :

Ménalque

«Pourquoi, Mopsus, puisque nous nous sommes rencontrés, tous deux experts,

toi à souffler dans un léger pipeau, moi à dire des vers,

ne nous asseyons-nous ici parmi les ormes mêlés aux coudriers?»

8. Ov., Met. I, 689 : Tum deus...

9. Ov., Met. I, 700-701 : restabat uerba referre/ et precibus spretis fugisse per auia nympham..., «lui restait à redire le discours [du dieu] / et que la nymphe, dédaignant ses prières, s'était enfuie... » 10. Ov., Met. I, 713-714 : Talia dicturus uidit Cyllenius omnes / succubuisse oculos.

11. Ov., Met. I, 557-559:

Cui deus : « At quoniam coniux mea non potes esse

arbor eris certe", dixit " mea. Semper habebunt

te coma, te citharae, te nostrae, laure, pharetrae...

«Alors le dieu : "Mais puisque tu ne peux, dit-il, être ma femme,

tu seras du moins mon arbre. Toujours te porteront,

toi, ma chevelure, toi, ma cithare, toi, laurier, mon carquois..." "

12. Todorov 1978, p. 17-18

13. Videau 2010, p. 309 sq.

14. Ov., Met. III, 638-675.

15. "Mais, dans les deux cas, au contraire des effets recherchés par Virgile au vers 210, le verbe au présent ne porte aucune trace du procès par lequel l'œil vient à briller, le corps de l'animal à se gonfler. L'hendiadyn cristis et auro ne complète plus un verbe mais un adjectif dénotant une qualité permanente, laudative, prcsignis ».

16. Arist., Po. 6, 50 a 7 sq.

17. Aron 1984, p. 9 : le terme literaturnost' est proposé par R. Jakobson dans La Poésie moderne russe, Prague, 1921, esquisse 1. La première attestation en français, en 1965, se trouve dans Todorov 1965, p. 37 : «R. Jakobson donna à cette idée sa formule définitive : 'L'objet de la science littéraire n'est pas la littérature, mais la littérarité (literaturnost'), c'est-à-dire ce qui fait d'une œuvre donnée une œuvre littéraire'».

18. Jakobson 1963, p. 213-222.

19. Mounin 1972, p. 151.

20. Aron 1984, p. 42 : «La lecture 'littéraire' implique la prise en compte totale, prioritaire de toute la surface du texte [...] littéralement et dans 'tous les sens' compatibles avec son dire [...] 
dans son rythme, sa ponctuation (au sens étroit et au sens large, métaphorique), ses divisions typographiques, voire sa graphie ».

21. Ov., Met. XI, 639-641.

22. Hom., Od. X, 302-306.

23. Les références homériques à la langue des dieux :

«Il est là-bas deux roches en surplomb contre lesquelles

roule la houle d'Amphitrite aux sombres yeux :

Les dieux bienheureux les nomment Roches planctes » (Od. XII, 59-61).

« [celui] que les dieux appellent Briarée et tous les hommes

Égéon, car Briarée l'emportait en force sur son père » (Il. I, 403-404).

« Devant la ville, il est une butte escarpée,

en retrait de la plaine, au pourtour accessible par différents côtés.

Cette butte, les hommes l'appellent Batiée,

et les Immortels le tertre de la bondissante Myrine » (Il. II, 811-814).

24. Bader 1993.

25. Gandon 2001, 2003 et 2006.

26. Frontisi-Ducroux, Vernant 1997, p. 212-213, cf. Plat., Phaedr. 255 c ; Arist., APo. 8, 419 b - 420 a.

27. Ov., Met. III, 356-361 :

«L'aperçoit, chassant vers ses filets les cerfs tremblants,

la nymphe sonore qui ne sait ni ne répondre à qui parle

ni parler la première, Écho, la résonnante.

Écho était alors de chair, elle n'était pas voix. Mais elle usait,

bavarde, de ses lèvres pareillement, [360]

ne savait, d'une suite de mots, redire que les derniers. »

28. Ov., Met. III, 379-394.

29. Le topos épique homérique du lever de l'Aurore trouve dans les Métamorphoses un certain nombre de variations dramatisées: voir l'épisode de Phaéthon(II), où l'Aurore devient personnage du récit à part entière et non plus simple protagoniste de la rythmique temporelle.

30. «Cette formule est entièrement construite sur des assonances vocaliques en [e], [o], [i], à la seule exception du verbe d'existence ou d'essence, erat à finale en [a]. La disposition embrassée des syntagmes parisyllabiques et homéotéleutes, en rime, pectore et in ore, encadrant en écho la répétition interne $\mathrm{du}$ nom de Procris, des deux côtés de la coupe penthémimère (erat)), représente l'obsession amoureuse, son inclusion au sein du sujet amoureux et sa proclamation sociale ».

31. Ov., Met. VII, $810 ; 812 ; 813 ; 822$.

32. Collart (éd.) 1954, X.

33. Ahl 1985, p. 51.

34. D'après Dupriez 1984, ad loc.

\section{RÉSUMÉS}

Le but de cet article est de montrer comment Ovide marque le texte des Métamorphoses comme texte, en suivant la trace des éléments qui mettent en perspective l'élaboration de la narration (jeux de genres et mise en abyme des récits, puis autonomisation de la description comme 
« morceau esthétique ») et la conception du signifiant dans l'œuvre et sa représentation dans le corps même du texte.

\section{INDEX}

nomsmotscles Argonautiques orphiques, Aristote, Eschyle, Homère, Ovide, Pacuvius (Marcus), Platon, Quintilien, Sophocle, Varron, Virgile

Mots-clés : conscience littéraire, description, énonciation, fonction métalinguistique, lecture littéraire, littérarité, mélange des genres, mise en abyme, mythe, narrateur, narration, récit enchâssé, signifiant, syntaxe, voix narrative

Keywords : literary awareness, description, enunciation, metalinguistic function, literary reading, literariness, mixed genres, mise en abyme, myth, narrator, narrative, story within a story, signifier, syntax, narrative voice

Index géographique : Arcadie 